\title{
Bernstein-type Theorems in Hypersurfaces with Constant Mean Curvature
}

\author{
MANFREDO P. DO CARMO ${ }^{1}$ and DETANG ZHOU ${ }^{2,3}$ \\ ${ }^{1}$ IMPA, Estrada Dona Castorina, 110 - Jardim Botanico, \\ 22460-320 - Rio de Janeiro, Brazil \\ ${ }^{2}$ Department of Mathematics, Shandong University, Jinan, Shandong 250100, China \\ ${ }^{3}$ Universidade Federal Fluminense, Instituto de Matemática - 24020-140 Niterói, Brazil
}

Manuscript received on February 21, 2000; accepted for publication on May 9, 2000;

contributed by MANFREDO DO CARMO*

\begin{abstract}
By using the nodal domains of some natural function arising in the study of hypersurfaces with constant mean curvature we obtain some Bernstein-type theorems.
\end{abstract}

Key words: Riemannian manifold, eigenvalue, hypersurface, mean curvature.

\section{INTRODUCTION}

The Bernstein theorem on minimal surfaces $x: M^{2} \rightarrow R^{3}$ in the Euclidean space $R^{3}$ states that if $x\left(M^{2}\right)$ is a graph over a plane $P$ of $R^{3}$ which is defined for all points of $P$ then $M^{2}$ is itself a plane. This beautiful result has been the basis of a large number of investigations on minimal surfaces. Among its generalizations is a theorem proved independently by (do Carmo \& Peng 1979) and (Fischer-Colbrie \& Schoen 1980) which states that if $M^{2}$ is complete and stable then it is a plane.

A generalization of this theorem for higher dimensions was obtained by (do Carmo-Peng 1980) as follows:

Theorem A. Let $x: M^{n} \rightarrow R^{n+1}$ be a minimal hypersurface. Assume that $M^{n}$ is stable, complete and that

$$
\lim _{R \rightarrow+\infty} \frac{\int_{B(R)}\|A\|^{2} d M}{R^{2+2 q}}=0, \quad q<\sqrt{2 / n} .
$$

Then $M^{n}$ is a hyperplane in $R^{n+1}$.

Here $\|A\|$ is the second fundamental form and $B(R)$ is a geodesic ball of radius ball $R$ centered at some fixed point in $M$.

\footnotetext{
* Member of the Academia Brasileira de Ciências

Correspondence to: Manfredo do Carmo

E-mail: manfredo@impa.br
} 
Theorem A has been recently extended to hypersurfaces with constant mean curvature. A crucial point is to replace $A$ by the traceless second fundamental form $\phi=-A+H I$; here $H$ is the mean curvature of $x: M^{n} \rightarrow R^{n+1}$. The precise statement is as follows:

Theorem B. (Alencar \& do Carmo 1994a). Let $x: M \rightarrow R^{n+1}, n \leq 5$ be a complete noncompact hypersurface with constant mean curvature $H$. Assume that $M$ is strongly stable (see definition in Section 1), and that

$$
\lim _{R \rightarrow+\infty} \frac{\int_{B(R)}\|\phi\|^{2} d M}{R^{2+2 q}}=0, \quad q<\frac{1}{6 n+1} .
$$

Then $M$ is a hyperplane in $R^{n+1}$.

In the present paper, we extend Theorem B in two directions. First we relax the growth condition on $P(R)=\int_{B(R)}|\phi|^{2} d M$ and extend Theorem B to this weaker condition. More precisely, we prove

THEOREM 1. Let $M^{n}$ be a strongly stable complete noncompact hypersurface of $R^{n+1}(n \leq 5)$ with constant mean curvature $H$. If $P(r) \leq C e^{\alpha H r}$, for some positive constants $C$, and $\alpha$, where $\alpha$ depends on $n$ given in the proof, then $M$ is a hyperplane.

Next we improve the dimension condition from $n \leq 5$ to $n \leq 6$ and prove

THEOREM 2. Let $M$ be a strongly stable complete noncompact hypersurface of $R^{n+1}(n \leq 6)$ with constant mean curvature $H$. Assume that

$$
\lim _{R \rightarrow+\infty} \frac{\int_{B(R)}\|\phi\|^{2} d M}{R^{2-2 / n}}=0 .
$$

Then $M$ is a hyperplane.

Theorem 1 is the main theorem of this paper and goes a long way towards getting rid of condition (0.1) in Theorem B. For its proof we need an auxiliary proposition that might be interesting by itself and states that the function $|\phi|$ on a hypersurface $M^{n}$ with constant mean curvature in $R^{n+1}$ has no bounded nodal domain.

\section{NOTATIONS AND PRELIMINARIES}

Let $M^{n}$ be a complete noncompact hypersurface in $R^{n+1}$. Fix $p \in M$ and choose a local unit normal field $N$. Define a linear map $A: T_{p} M \rightarrow T_{p} M$ by

$$
\langle A X, Y\rangle=\left\langle\bar{\nabla}_{X} Y, N\right\rangle
$$

where $X, Y$ are the tangent vector fields and $\bar{\nabla}$ is the standard connection on $R^{n+1}$. The map $A$ can be diagonalized, i.e., there exists a tangent basis $\left\{e_{1}, e_{2}, \cdots, e_{n}\right\}$ such that $A e_{i}=k_{i} e_{i}, i=1,2, \cdots, n$. We then define the mean curvature $H:=\frac{1}{n} \sum_{i=1}^{n} k_{i}$ and the square of the second fundamental form $|A|^{2}:=\sum_{i=1}^{n} k_{i}^{2}$. It is well known that the above objects are independent of the choices made. 
If $M$ is minimal $(H=0)$, we say $M$ is stable if for all piecewise smooth functions $f: M \rightarrow R$ with compact support, we have that

$$
\int_{M}|\nabla f|^{2} d M \geq \int_{M}|A|^{2} f^{2} d M
$$

here $\nabla f$ is the gradient of $f$ in the induced metric.

The notion of stability has been extended to hypersurfaces with constant mean curvature as follows: $M$ is said to be strongly stable if (1.1) holds for all piecewise smooth functions $f: M \rightarrow R$ with compact support. $M$ is said to be weakly stable if (1.1) holds for all piecewise smooth functions $f: M \rightarrow R$ with compact support and $\int_{M} f=0$.

Let $x: M^{n} \rightarrow \bar{M}^{n+1}$ be an isometric immersion of a complete, noncompact Riemannian $n$ dimensional manifold $M^{n}$ into an oriented, complete, Riemannian $(n+1)$-dimensional manifold, $N$ a smooth unit normal field along $M$, and $\overline{\operatorname{Ric}}(N)$ the value of the Ricci curvature of $\bar{M}^{n+1}$ in the vector $N$. Here $\overline{\operatorname{Ric}}(N)=\sum_{i=1}^{n} K\left(e_{i} \wedge N\right)$ (this is different from the normalized one). The Morse index ind $M$ of $\mathrm{M}$ is defined as follows. Let $L$ be the second order differential operator on $M$ given by

$$
L=\Delta+|A|^{2}+\overline{\operatorname{Ric}}(N) .
$$

Associated to $L$ is the quadratic form

$$
I(f)=-\int_{M} f L f d M,
$$

defined on the vector space of functions $f$ on $M$ that have support on a compact domain $K \subset M$. For each such $K$, define the index $\operatorname{ind}_{L} K$ of $L$ in $K$ as the maximal dimension of a subspace where $I$ is negative definite. The index ind $M$ of $\mathrm{L}$ in $M$ is the number defined by

$$
\text { ind } M=\sup _{K \subset M} \operatorname{ind}_{L} K
$$

where the supremum is taken over all compact domains $K \subset M$. It is well known that ind $(M) \leq 1$, if $M$ is weakly stable(see, for example, (Fischer-Colbrie 1985)).

In what follows we always assume that $M$ is a hypersurface in $R^{n+1}$ with constant mean curvature $H$. To study the hypersufaces with constant mean curvature, it is convenient to modify slightly the second fundamental form and to introduce a new linear map $\phi: T_{p} M \rightarrow T_{p} M$ by

$$
\langle\phi X, Y\rangle=-\langle A X, Y\rangle+H\langle X, Y\rangle .
$$

$\phi$ can also be diagonalized as:

$$
\phi e_{i}=\mu_{i} e_{i}
$$

It is easily checked that $\operatorname{tr} \phi=0$, and

$$
|\phi|^{2}:=\sum_{i=1}^{n} \mu_{i}^{2}=\frac{1}{2 n} \sum_{i, j}\left(k_{i}-k_{j}\right)^{2} .
$$


Thus $|\phi|^{2}$ measures how far $M$ is from being totally umbilic. For the rest of this section we follow (Alencar $\&$ do Carmo 1994a). Choosing an orthonormal principal frame $\left\{e_{i}\right\}$, we can write

$$
\frac{1}{2} \Delta|\phi|^{2}=\sum_{i, j, l} \phi_{i j l}^{2}+\sum_{i} \mu_{i}(\operatorname{tr} \phi)_{i i}+\frac{1}{2} \sum_{i, j} R_{i j i j}\left(\mu_{i}-\mu_{j}\right)^{2}
$$

where $\phi_{i j l}$ are components of the covariant derivative of the tensor $\phi$, and $R_{i j i j}$ is the sectional curvature of the plane $\left\{e_{i}, e_{j}\right\}$. By Gauss formula, we conclude that

$$
\begin{aligned}
\frac{1}{2} \sum_{i, j} R_{i j i j}\left(\mu_{i}-\mu_{j}\right)^{2}= & \frac{1}{2} \sum_{i, j} \mu_{i} \mu_{j}\left(\mu_{i}-\mu_{j}\right)^{2} \\
& -\frac{H}{2} \sum_{i, j}\left(\mu_{i}+\mu_{j}\right)\left(\mu_{i}-\mu_{j}\right)^{2}+\frac{H^{2}}{2} \sum_{i, j}\left(\mu_{i}-\mu_{j}\right)^{2} .
\end{aligned}
$$

Since $\sum \mu_{i}=0$, it is easy to check that:

$$
\begin{aligned}
\sum_{i, j}\left(\mu_{i}-\mu_{j}\right)^{2} & =2 n|\phi|^{2}, \\
\sum_{i, j}\left(\mu_{i}+\mu_{j}\right)\left(\mu_{i}-\mu_{j}\right)^{2} & =2 n \sum_{i} \mu_{i}^{3}, \\
\sum_{i, j} \mu_{i} \mu_{j}\left(\mu_{i}-\mu_{j}\right)^{2} & =-2|\phi|^{4} .
\end{aligned}
$$

From the above, it follows that

$$
\frac{1}{2} \Delta|\phi|^{2}=|\phi| \Delta|\phi|+|\nabla| \phi||^{2}=\sum_{i, j, l} \phi_{i j l}^{2}-|\phi|^{4}-n H \sum_{i} \mu_{i}^{3}+n H^{2}|\phi|^{2} .
$$

In this case it follows by (do Carmo \& Peng 1980 (2.3), (2.4)) that

$$
\sum_{i, j, l} \phi_{i j l}^{2} \geq \frac{2}{n}|\nabla| \phi||^{2}+|\nabla| \phi||^{2}
$$

By using a lemma of Okumura (see (Alencar \& do Carmo 1994b) for a proof), we have

$$
\sum_{i} \mu_{i}^{3} \leq \frac{n-2}{\sqrt{n(n-1)}}|\phi|^{3}
$$

So we have finally

$$
|\phi| \Delta|\phi|+|\phi|^{4}+\frac{n(n-2)}{\sqrt{n(n-1)}} H|\phi|^{3}-n H^{2}|\phi|^{2} \geq\left.\frac{2}{n}|\nabla| \phi\right|^{2} .
$$




\section{A RESULT ON NODAL DOMAINS}

In this section we prove a result on the nodal domains of $|\phi|$ which will be needed in our proof of main theorems. We first need to recall the definition of nodal domains.

Definition. An open domain $D$ is called the nodal domain of function $f$ if $f(x) \neq 0$ for $x \in$ int $D$ and vanishes on the boundary of $\partial D$. We denote by $N(f)$ the number of disjoint bounded nodal domains of $f$.

Now we have the following lemma which follows directly from Proposition 2.2 below. We are indebted to the referee who provided its proof and corrected a mistake in our original version. LEMMA 2.1. Let $M$ be a hypersurface in $R^{n+1}$ with constant mean curvature $H$. Then

$$
N(|\phi|)=0
$$

Proof. Let $\varphi(u)=u^{2}+\frac{n(n-2)}{\sqrt{n(n-1)}} H u-n H^{2}$. Then from (1.5), with $|\phi|=u$, and Proposition 2.2 below the lemma follows.

Proposition 2.2. Let $(M, g)$ be Riemannian manifold and $u \geq 0$ be a continuous function satisfying the following inequality of Simons' type in the distribution sense

$$
u^{2} \varphi(u) \geq a|\nabla u|_{g}^{2}-u \Delta_{g} u
$$

where $a>0$ is a constant and $\varphi$ is a continuous function on $R$.

Then $u$ has no relatively compact nodal domain.

Proof. Suppose that $u$ admits a relatively compact nodal domain $D$. Write $q:=\varphi(u)$ and $v:=\log u$ on $D$. Thus (2.2) can be written as

$$
q \geq a|\nabla v|_{g}^{2}-\Delta_{g} v-|\nabla v|_{g}^{2}
$$

Then for any Lipschitz function $f$ with support in $D$ and vanishing at $\partial D$, we have

$$
\int_{D}\left(|\nabla f|^{2}-q f^{2}\right) \leq-a \int_{D} f^{2}|\nabla v|^{2}+\int_{D}|\nabla f-f \nabla v|^{2} .
$$

Let $f=w u$, for some function $w$ to be determined. We obtain

$$
\int_{D}\left(|\nabla f|^{2}-q f^{2}\right) \leq-a \int_{D} w^{2}|\nabla u|^{2}+\int_{D} u^{2}|\nabla w|^{2} .
$$

For all $b$ such that $U / 2 \leq b \leq U$, where $U:=\sup _{D} u$, we set

$$
w_{b}(x)= \begin{cases}b & \text { if } u(x) \leq b \\ u(x), & \text { if } u(x)>b\end{cases}
$$


Denote $D_{+}$(resp. $D_{-}$) the set of points in $D$ with $u(x) \geq b$ (resp. $\left.u(x) \leq b\right)$. A simple calculation leads to

$$
\int_{D}\left(|\nabla f|^{2}-q f^{2}\right) \leq \int_{D_{+}} u^{2}|\nabla u|^{2}-\frac{a U^{2}}{4} \int_{D}|\nabla u|^{2} .
$$

When $b$ goes to $U$, the first term of right hand side tends to 0 (because $|\nabla u|^{2}$ is integrable), while the second term is fixed. It follows that $\int_{D}\left(|\nabla f|^{2}-q f^{2}\right)<0$ for all functions $f=w_{b} u$, when $b$ is close to $U$. These functions $w_{b}$ form an infinite dimensional vector which leads to a contradiction to the fact that $D$ is relatively compact and $q$ is continuous.

\section{BERNSTEIN-TYPE THEOREMS}

Before proving our main theorem, we need an auxiliary proposition. Set

$$
P(r)=\int_{B(r)}|\phi|^{2} d v .
$$

Proposition 3.1. Let $M$ be a complete noncompact hypersurface of $R^{n+1}(n \leq 5)$ with constant mean curvature $H(H \neq 0)$ and finite index. Assume that $P(r) \leq C e^{\alpha H r}$ for some positive constants $C$, and $\alpha$, where $\alpha$ is a constant that can expressed explicitly in terms of $n$. Then $\int_{M}|\phi|^{2}<+\infty$.

Our Theorem 1 is a corollary of the above proposition. It is a combination of the proposition and theorems in (Alencar \& do Carmo 1994a) and (do Carmo \& Peng 1980). Before proving Proposition 3.1 we give the proof of Theorem 1.

Proof of TheOrem 1. To prove the conclusion of Theorem 1 we only need to show that $H=0$ by Theorem A. Otherwise $H \neq 0$, and by Proposition 3.1 we know that $\int_{M}|\phi|^{2}<+\infty$. This is impossible by Theorem B. Thus the proof is complete.

We now prove the proposition:

Proof of Proposition 3.1. Introduce $f|\phi|^{q+1}$ in the stability inequality (1.1). It has been shown in (Alencar \& do Carmo 1994a) that for all $\epsilon>0$,

$$
\int_{M} f^{2}|\phi|^{2+2 q}\left[A|\phi|^{2}-B|\phi|+C\right] \leq D \int_{M}|\phi|^{2 q+2}|\nabla f|^{2},
$$

where

$$
\begin{aligned}
& A=1-(1+q+\epsilon)\left(\frac{2}{n}+q\right)^{-1} q, \\
& B=(1+q+\epsilon)\left(\frac{2}{n}+q\right)^{-1}(1+q) \frac{n(n-2)}{\sqrt{n(n-1)}} H, \\
& C=\left(1+(1+q+\epsilon)\left(\frac{2}{n}+q\right)^{-1}(2+q)\right) n H^{2}, \\
& D=(1+q+\epsilon)\left(\frac{2}{n}+q\right)^{-1}+1+\frac{1+q}{\epsilon} .
\end{aligned}
$$


If $M$ has finite index then it is stable outside some ball $B(R)$. In (3.1), we choose $q=0$; then $A=1$ and

$$
\begin{aligned}
B & =(1+\epsilon) \frac{n}{2} \frac{n(n-2)}{\sqrt{n(n-1)}} H, \\
C & =[1+n(1+\epsilon)] n H^{2}, \\
D & =\frac{n(1+\epsilon)}{2}+1+\frac{1}{\epsilon} .
\end{aligned}
$$

So in this case we have

$$
\begin{aligned}
A|\phi|^{2}-B|\phi|+C= & \left(|\phi|-\frac{B}{2}\right)^{2}+\frac{4 C-B^{2}}{4} \\
\geq & \frac{4 C-B^{2}}{4} \\
= & -\left[n^{2}(n-2)^{2} \epsilon^{2}+2 n\left(n^{3}-4 n^{2}-4 n+8\right) \epsilon\right. \\
& \left.\quad+n^{4}-4 n^{3}-12 n^{2}+16\right] H^{2} .
\end{aligned}
$$

It can be checked that when $n \leq 5$, we can find sufficiently small $\epsilon>0$ such that $4 C-B^{2}>0$. So there exists a constant $\beta$ which can expressed in terms of $n$ such that

$$
H^{2} \int_{M \backslash B(R)} f^{2}|\phi|^{2} \leq \beta^{-2} \int_{M \backslash B(R)}|\phi|^{2}|\nabla f|^{2},
$$

for any piecewise smooth function $f$ with compact support in $M \backslash B(R)$. Then

$$
\beta^{2} H^{2} \leq \frac{\int_{M \backslash B(R)}|\phi|^{2}|\nabla f|^{2}}{\int_{M \backslash B(R)} f^{2}|\phi|^{2}} .
$$

We claim that we can choose $R$ large enough such that $P^{\prime}(r)>0$ for all $r>R$. Otherwise we can find two positive constants $r_{1}<r_{2}$ such that $|\phi|(x)=0$ when $x \in \partial B\left(r_{i}\right)$. Thus $B\left(r_{2}\right) \backslash \overline{B\left(r_{1}\right)}$ contains a nodal domain and this contradicts Lemma 2.1.

Assume for the sake of the contradiction that $P(+\infty)=+\infty$. Then from our oscillation theorem in (do Carmo \& Zhou 1999 Theorem 2.1) we have that for any $\lambda>\frac{\alpha^{2} H^{2}}{4}$ we can find $x(t)$ which is not identically zero and is an oscillatory solution of

$$
\left[P^{\prime}(t) x^{\prime}(t)\right]^{\prime}+\lambda P^{\prime}(t) x(t)=0
$$

Choose $f(x)=x(r(x))$ where $r(x)$ is the distance function to some fixed point in $M$. We can find $T_{1}$ and $T_{2}$, such that $T_{2}>T_{1}>R$ and $x\left(T_{1}\right)=x\left(T_{2}\right)=0, x(t)>0$ for all $t \in\left(T_{1}, T_{2}\right)$. Now choose $\lambda=\left(\frac{\alpha^{2}}{4}+\delta\right) H^{2}$, where $\delta>0$ is a constant such that $\beta^{2}-\delta>0$ and set $\alpha<2 \sqrt{\beta^{2}-\delta}$. It follows that

$$
\beta^{2} H^{2} \leq \frac{-\int_{T_{1}}^{T_{2}}\left[P^{\prime}(r) x^{\prime}(r)\right]^{\prime} x(r) d r}{\int_{T_{1}}^{T_{2}} P^{\prime}(r) x^{2}(r) d r}=\lambda<\beta^{2} H^{2} .
$$

This is a contradiction which shows our conclusion. 
We now give the proof of Theorem 2:

Proof of Theorem 2. We can assume that $H \neq 0$; otherwise from (do Carmo \& Peng 1980) the theorem holds. Notice that in (3.1)

$$
\begin{aligned}
B^{2}-4 A C= & \frac{n H^{2}}{(n-1)(2+n q)^{2}}\left\{n^{4} q^{4}+2 n^{4}(\epsilon+2) q^{3}\right. \\
& \quad+n^{2}\left(n^{2} \epsilon^{2}+6 n^{2} \epsilon+6 n^{2}-16 n+16\right) q^{2} \\
& +2 n\left[n^{3} \epsilon^{2}+\left(3 n^{2}-8 n+8\right) n \epsilon+2\left(n^{3}-4 n^{2}-4 n+8\right)\right] q \\
& \left.+\left[n^{2}(n-2)^{2} \epsilon^{2}+2 n\left(n^{3}-4 n^{2}-4 n+8\right) \epsilon+n^{4}-4 n^{3}-12 n^{2}+16\right]\right\} .
\end{aligned}
$$

Consider the terms without $\epsilon$ in the large bracket:

$$
\begin{aligned}
g(n, q):= & n^{4} q^{4}+4 n^{4} q^{3}+n^{2}\left(6 n^{2}-16 n+16\right) q^{2} \\
& +4 n\left(n^{3}-4 n^{2}-4 n+8\right) q+n^{4}-4 n^{3}-12 n^{2}+16
\end{aligned}
$$

Then, by choosing $q=-\frac{1}{n}$,

$$
\begin{aligned}
g\left(n,-\frac{1}{n}\right)= & 1-4 n+6 n^{2}-16 n+16-4 n^{3}+16 n^{2} \\
& \quad+16 n-32+n^{4}-4 n^{3}-12 n^{2}+16 \\
= & n^{4}-8 n^{3}+10 n^{2}-4 n+1 \\
= & (n-1)\left(n^{3}-7 n^{2}+3 n-1\right) .
\end{aligned}
$$

It is easy to see that $g\left(n,-\frac{1}{n}\right)<0$ when $n \leq 6$. Thus we can always choose $\epsilon$ sufficient small such that $B^{2}-4 A C<0$. Notice that our choice of $q=-\frac{1}{n}$ makes $A>0$. By using Young's inequality in $(3.1)$

$$
\int_{M} f^{2}|\phi|^{2+2 q}\left[A|\phi|^{2}-B|\phi|+C\right] \leq \delta \int_{M}|\phi|^{2 q+4} f^{2}+\beta_{1} \int_{M} \frac{|\phi|^{2}|\nabla f|^{2(1+q)}}{f^{2 q}},
$$

where $\beta_{1}>0$ is a constant (depending on $n, \epsilon$, and $q$ ) and $\delta>0$ can be chosen arbitrarily small. Now set $\bar{A}=A-\delta$ and choose $\delta$ small enough so that $B^{2}-4 \bar{A} C>0$ and $\bar{A}>0$. It follows from (3.7) that

$$
\int_{M} f^{2}|\phi|^{2+2 q} \leq \beta_{2} \int_{M} \frac{|\phi|^{2}|\nabla f|^{2(1+q)}}{f^{2 q}} .
$$

Writing $f=h^{1+q}$, we have

$$
\int_{M} f^{2+2 q}|\phi|^{2+2 q} \leq \beta_{3} \int_{M}|\phi|^{2}|\nabla f|^{2+2 q} .
$$

where $\beta_{3}$ is a constant depending only on $n, \epsilon$. The rest of the proof follows exactly as in (do Carmo \& Peng 1980), and we find that $H=0$, a contradiction. 


\section{SOME FURTHER RESULTS}

In this section we want to give some further related results. Using the eigenvalue estimate in (do Carmo \& Zhou 1999) we can get an index estimate for hypersurfaces with nonzero constant mean curvature.

Define $\alpha(M):=\limsup _{r \rightarrow+\infty} \frac{\log V(r)}{r}$ where $V(r)$ is the volume of geodesic ball $B(r)$. It is easy to see that $\alpha(M)=0$ if $M$ has polynomial volume growth.

THEOREM 4.1. If $M$ is complete noncompact hypersurface in $R^{n+1}$ with nonzero constant mean curvature $H$ and $\alpha(M)<2 \sqrt{n} H$, then ind $(M)=+\infty$.

In order to prove this Theorem we need to use the eigenvalue estimate theorem proved by the authors in (do Carmo \& Zhou 1999) which is now restated as follows.

THEOREM. Let $M$ be a complete noncompact Riemannian manifold with infinite volume and $\Omega$ be an arbitrary compact subset of $M$. Then

$$
\lambda_{1}(M \backslash \Omega) \leq \frac{\alpha^{2}}{4} .
$$

Proof of Theorem 4.1. It suffices to prove that for any natural number $N$ we can find piecewise smooth functions $f_{1}, f_{2}, \cdots, f_{N}$ with compact supports such that $\operatorname{supp}\left(f_{i}\right)$ are disjoint and $I\left(f_{i}\right)<$ 0 .

Note that from (Frensel, 1996) the volume of $M$ is infinite, so from the Theorem we have:

$$
\lambda_{1}(M \backslash \Omega) \leq \frac{\alpha^{2}}{4}<n H^{2},
$$

for any compact set $\Omega$ in $M$. So we can find a compact domain $D_{1}$ such that $\lambda_{1}\left(D_{1}\right) \leq \frac{\alpha^{2}}{4}<n H^{2}$. We also have $\lambda_{1}\left(M \backslash D_{1}\right) \leq \frac{\alpha^{2}}{4}<n H^{2}$. So we can find again a compact domain $D_{2} \subset M \backslash D_{1}$ such that $\lambda_{1}\left(D_{2}\right) \leq \frac{\alpha^{2}}{4}<n H^{2}$. and $\lambda_{1}\left(M \backslash\left(D_{1} \cup D_{2}\right)\right) \leq \frac{\alpha^{2}}{4}<n H^{2}$. Repeating this procedure, we can find disjoint compact domains $D_{1}, D_{2}, \cdots, D_{N}$, such that $\lambda_{1}\left(D_{i}\right)<n H^{2}$.

Let $\varphi_{i}$ be the positive first eigenfunction of $\Delta_{M}$ on $D_{i}$, i.e.: $\Delta \varphi_{i}=\lambda_{i}\left(D_{i}\right) \varphi_{i}$ in $D_{i}$ and $\varphi_{i}=0$ on $\partial D_{i}$. We now define $f_{i}(x):=\varphi_{i}(x)$ for $x \in D_{i}$ and $f_{i}(x) \equiv 0$ for $x \in M \backslash D_{i}$. So

$$
\int_{M}\left|\nabla f_{i}\right|^{2}=\lambda_{1}\left(D_{i}\right) \int_{M} f_{i}^{2}<n H^{2} \int_{M} f_{i}^{2} \leq \int_{M}|A|^{2} f_{i}^{2} .
$$

Thus $I\left(f_{i}, f_{i}\right)<0$ for $i=1,2, \cdots, N$. This shows that ind $(M) \geq N$, for any $N$. So ind $(M)=$ $+\infty$.

The following is an easy consequence of Theorem 4.1 .

COROLLARY 4.2. If $M$ is complete noncompact hypersurface with nonzero constant mean curvature $H$ and polynomial volume growth, then $\operatorname{ind}(M)=+\infty$. In particular, $\operatorname{ind}(M)=+\infty$, when $M=S^{k} \times R^{n-k}$ with the standard metric; here $S^{k}$ is a k-dimensional sphere in $R^{k+1}$. 


\section{ACKNOWLEDGMENTS}

This work was done while the second author was visiting Instituto de Matemática Pura e Aplicada by an associate membership scheme of TWAS-IMPA. He wishes to thank these institutions for support and IMPA for the hospitality. The authors dedicate this paper to the memory of Carlos Chagas Filho. This work was supported partially by NNSFC, CNPq and TWAS-IMPA membership.

\section{REFERENCES}

Alencar H \& do Carmo MP. 1994a. Hypersurfaces with constant mean curvature in space forms. An Acad Bras Ci 66: 265-274.

Alencar H \& do Carmo MP. 1994b. Hypersurfaces with constant mean curvature in spheres. Proc Amer Math Soc 120: 1223-1229.

do Carmo MP \& Peng CK. 1979. Stable complete minimal surfaces in $R^{3}$ are planes. Bull Amer Math Soc N.S. 1: 903-906.

do Carmo MP \& Peng CK. 1980. Stable complete minimal hypersurfaces. Proc of the 1980 Beijing Symp. Chern SS \& Wentsun W (Eds.), Gordan and Breach Science Pub., 1349-1358.

do Carmo MP \& Zhou D. 1999. Eigenvalue Estimate on Complete Noncompact Riemannian Manifolds and Application. Trans Amer Math Soc 351: 1391-1401.

Fisher-Colbrie D. 1985. On complete minimal surfaces with finite Morse index in three-manifolds. Invent Math 82: 121-132.

Fisher-Colbrie D \& Schoen R. 1980. The structure of complete stable minimal surfaces in 3-manifolds of nonnegative curvature. Comm Pure Appl Math 33: 199-211.

FRENSEL KR. 1996. Stable complete surfaces with constant mean curvature. Bol Soc Bras Mat 27: 129-144. 